\title{
Quality of experience
}

\section{1-Metrics and performance evaluation}

\author{
Markus Fiedler • Helmut Hlavacs • Klaus Hackbarth • \\ Patrik Arlos
}

(C) Institut TELECOM and Springer-Verlag 2010

During the recent years, we have observed a significant growth in offers, varieties, and uses of network-based services. This was amongst others enabled by a growing multitude of access networks combined with usage-friendly pricing schemes. However, the conditions for providing this access vary between, and also within, regions of this planet. As a consequence, potential users become victims of the "Digital Divide", therefore being excluded from beneficial use of Information Communication Technology (ICT) services. Consequently, socio-economic-technical aspects and scenarios need to be researched in order to reduce the "Digital Divide".

Research within ICT is still quite technology oriented, and often neglects the important window towards the end user who wants to "get the most out of it" when using and paying for services. In particular, in this special issue, we address Quality of Experience (QoE) on one side and-

\author{
M. Fiedler $(\bowtie)$ \\ Blekinge Institute of Technology, \\ Karlskrona, Sweden \\ e-mail: markus.fiedler@bth.se \\ H. Hlavacs \\ University of Vienna, \\ Vienna, Austria \\ e-mail: helmut.hlavacs@univie.ac.at \\ K. Hackbarth \\ University of Cantabria, \\ Santander, Spain \\ e-mail: klaus@tlmat.unican.es \\ P. Arlos \\ Blekinge Institute of Technology, \\ Karlskrona, Sweden \\ e-mail: patrik.arlos@bth.se
}

mostly economical - terms of use on the other side. If a user does not feel that (s)he is getting "value for money", i.e., good-enough QoE for what (s)he is paying for, (s)he might be ready to abandon the particular service provider and/or network operator in question. We are thus facing a quality- and economy-related type of "Digital Divide". Obviously, quality and economy form important enablers for successful service provisioning, which require a careful investigation and evaluation of behaviors, conditions, and capabilities at the border between user and technology.

In this context, the notion and topic of QoE increasingly attracts the attention of manufacturers, operators, and researchers. It links user perception and expectations on one side and technical Quality of Service parameters, management, pricing schemes, etc., on the other side. Such links are needed in order to balance user satisfaction and economic aspects of service provisioning. However, the notion of QoE as such is not without controversy. Technicians, used to a world of objective and clearly definable parameters, tend to fear the subjective, somehow fuzzy parts associated with end-user perception. Vice versa, customer relationship and marketing departments might find themselves uncomfortable with technical parameters which might not reflect the user perception in some tense situations. Nevertheless, appearance and utility of a networked service depend on the underlying technical solutions and their performance. Furthermore, legal and pricing issues need to be addressed, which also implies resource trading and dimensioning.

The first volume of this Special Issue on Quality of Experience and Socio-Economic Issues of Network-Based Services is devoted to the topic of QoE metrics and performance evaluation, ranging from the application to the network level. 
The first paper "Automated Qualitative Assessment of Multi-Modal Distortions in Digital Images Based on GLZ" by Andrzej Głowacz, Michał Grega, Przemysław Gwiazda, Lucjan Janowski, Mikołaj Leszczuk, Piotr Romaniak, and Simon Pietro Romano addresses the issue of replacing time-consuming and costly psychophysical experiments by automatic image quality assessment. A large set of subjective quality scores created the base for the evaluation of the impact of seven types of image distortions, in particular contrast; blur; granularity; noise; and distortions of geometry, color and gamma, respectively. From these scores, mapping models were derived with aid of the regression method Generalized Linear Model (GLZ) and validated in terms of Most Probable Opinion Score (MPOS) differences between test users and predictions. It turned out that, in most cases, MPOS differences were small, indicating a satisfactory match provided by the GLZbased metrics.

The second paper "An Integrated Framework of HoQ \& AHP for QoE Improvement of Network-based ASP Services" by Dohoon Kim addresses the House of Quality (HoQ) approach. HoQ is applied by Application Service Providers for successfully implementing, customizing and managing Internet-based e-business transactions. Targeting good QoE, the Analytical Hierarchy Process was used to combine and trade between the Voice of Customers with related Service Attributes on one hand and the Voice of Engineers with related Engineering Characteristics on the other hand. The proposed method allows for substituting difficult-to-achieve qualitative measurements by quantitative and objective estimations, and provides thus strategic directions for QoE improvements.

The third paper "Optimal Server Bandwidth for Mobile Video on Demand" by Shelley Buchinger and Helmut Hlavacs targets a reasonable trade-off between bandwidth consumption for video-on-demand delivery and user perception. To this end, the recently proposed Hierarchical Patching scheme that allows users to catch up and hook into an ongoing multicast transmission was extended by a low-start phase. During the latter, the start of the movie is encoded with a lower bit rate than the rest of the movie. Through argumentation involving probabilities of video segment transmittal, it was shown analytically that the proposed method minimizes the server bandwidth to a significant extent. Experiments with human observers revealed that a short but strongly bandwidth-reduced start-up phase is preferable from the user's point of view.
The fourth paper "Quality of Experience from User and Network Perspectives" by Junaid Shaikh, Markus Fiedler and Denis Collange brings together users' views on application performance and possible evidence of user (dis-)satisfaction as seen from the operator's point of view. To this aim, it identified mostly non-linear functions that relate response times and perceived throughputs to loss ratios and the impact of those three parameters on user ratings of response times taken from a test bed. Taking the network point-of-view, it quantified the non-linear effect of data loss and achieved throughput on session volumes from operator measurements in a public DSL network. From the comparison of both sets of approximation formulae, clear indications that "happy users surf more" were obtained, while average user-related waiting times were found to be almost invariant to the QoE.

The fifth paper "Towards Real-Time Anomalies Monitoring for QoE Indicators" by Frédéric Guyard and Sergio Beker faces the challenge that, in order to satisfy emerging QoE-based Service Level Agreements, network states that are affecting the user perception in a negative way need to be discovered and characterized. The latter has to happen in a reliable way even in face of highly complex state spaces that stem from the current multi-user multi-service networking environment. The proposed anomaly detection method builds upon the representation of QoE indicators as a time series. Upon reduction of the state space by employing entropy time series, Singular Spectrum Analysis, using a combination of geometric and statistical methods, was applied. The capability of the proposed method to discern anomalies from normal behavior was demonstrated through simulation.

The sixth and last paper "Revisiting FAST TCP Fairness" by Tuan A. Trinh, Balász Sonkoly, and Sándor Molnár investigates the impact of protocol parameter settings for FAST TCP in terms of fairness and thus of QoE. Time plots obtained from ns-2 simulations revealed that proper parameter settings allowed for robust fairness in combinations with HighSpeed TCP and Scalable TCP, respectively. Nevertheless, the paper highlights challenges of the fairness such as complex network environments with multiple congested links and the dynamic behavior towards a potential equilibrium. Based on these observations, it proposes research into dynamic sensitive fairness metrics for performance evaluation of transport protocols for next generation high bandwidth-delay product networks.

We would like to thank the authors for their efforts, as well as the reviewers. 